\title{
A Methodology to Validate MRI/SPECT Registration Methods Using Realistic Simulated SPECT Data
}

\author{
Christophe Grova ${ }^{1}$, Arnaud Biraben ${ }^{1}$, Jean-Marie Scarabin ${ }^{1}$, Pierre Jannin ${ }^{1}$, \\ Irène Buvat ${ }^{2}$, Habib Benali ${ }^{2}$, and Bernard Gibaud ${ }^{1}$ \\ 1 Laboratoire IDM, Faculté de Médecine, Université de Rennes 1, France \\ \{christophe.grova, arnaud.biraben, jean-marie.scarabin, \\ pierre.jannin, bernard.gibaud\} @univ-rennes1.fr, \\ http://idm.univ-rennes1.fr \\ 2 INSERM U494, CHU Pitié Salpétrière, Paris \\ \{irene.buvat, habib.benali\}@imed.jussieu.fr
}

\begin{abstract}
We present a method to validate MRI/SPECT registration methods based on a set of computer-generated SPECT data. The data set was produced through Monte Carlo simulations from an attenuation map and an activity map derived from a manually labeled T1-weighted MRI data set.

Our approach intrinsically provides a gold standard to assess MRI/SPECT registration methods. It was successfully applied to the comparison of four registration methods based on similarity measurements: Mutual Information, Normalised Mutual Information, Correlation Ratio and Woods Criterion.
\end{abstract}

\section{Introduction}

Multimodal data fusion has a strong potential to improve diagnosis and treatment preparation in many fields making intensive use of medical images. The accuracy of registration between imaging modalities is central in the technique. That is why the selection of appropriate registration methods should take each clinical context into account (e.g. ictal epileptic state) in order to thoroughly investigate whether the basic assumptions underlying each method (e.g. nature of similarities) are met.

Registration methods have been widely compared and validated for CT, MRI and PET data, particularly in the Retrospective Registration Evaluation Project (RREP) led by Vanderbilt University [1]. However, only few references are specifically geared toward validating SPECT/MRI registration methods 2] 3. In most cases the methods validated for PET/MRI registration are simply applied to register SPECT data 1] [4].

But in order to validate a method, the precision and accuracy of a registration technique should be assessed by means of a gold standard. The latter provides a reference geometric transformation with which registration methods 
can be assessed. Classically, gold standards are provided by fiducial markers [2], stereotactic frames [1] [4] [5] or mean geometric transformation computed from several registration results $[3$. But this type of evaluation is limited by the intrinsic accuracy and precision of the registration method used to compute the gold standard. Indeed, the method to be validated cannot yield better performances than the gold standard registration method itself. We suggest that a "perfect" gold standard can be found by using simulated data to validate methods. The main drawback of simulated data is that they are generally too far from clinical reality, possibly causing validation results to be biased by too auspicious test conditions.

We present a validation method based on realistic SPECT simulations to study and compare SPECT/MRI registration methods. We used Monte Carlo simulations to create normal SPECT from an MRI data set. Simulated data were then used to study four MRI/SPECT registration methods based on similarity measurements: Mutual Information (MI) [7] [], Normalised Mutual Information (NMI) 6], Correlation Ratio (CR) 9] and Woods Criterion (WC) 4].

\section{Material and Methods}

\subsection{SPECT Model Construction}

Our method is based on the simulation of realistic SPECT data sets from a 3D T1-weighted MRI data set. By constructing simulated SPECT data from an MRI data set, we can be certain that both data sets are perfectly aligned. As a result, our method provides a gold standard for validation. We constructed realistic SPECT data sets by simulating physical processes including both single photon propagation (e.g. Compton scatter, tissue attenuation) and acquisition procedures (e.g. collimator and detector response), using Monte Carlo techniques. Our simulations were performed with the Photon History Generator (PHG) software package created by the Simset team (Simulation System for Emission Tomography 1 ) from Washington University [10. Actually, PHG simulates SPECT data from a theoretical model of brain perfusion. A brain tissue attenuation map and a radioactive tracer activity map are used to build the perfusion model from a high resolution MRI data set.

Spatial Model of Radiotracer Distribution Our spatial distribution model was based on Zubal's head phantom, which consists of sixty three anatomical entities manually segmented and labeled on a normal T1-weighted MRI data set [11. These entities were classified into seven different classes (conjunctive tissue, water, brain, bone, muscle, fat and blood), from which the attenuation map was derived.

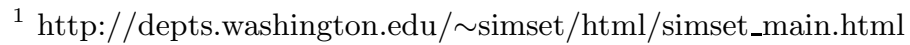


Brain Perfusion Model Using labeled entities of Zubal's phantom, we, along with clinicians, selected eight anatomical structures likely to fix the radioactive tracer (HMPAO- ${ }^{99 m} \mathrm{Tc}$ ) differently: external cortex, occipital lobes, pons, white matter, cerebellum, insula, gray nuclei and caudate nuclei. For each anatomical structure, we extracted eight binary masks from the labeled MRI. Our measurements of normal brain perfusion were performed on the SPECT template provided by the Statistical Parametric Mapping (SPM) software packag£2. This template was generated by Barnden [12] by averaging 22 normal SPECT data sets after spatial normalization. Perfusion measurements were performed on the SPECT template using the eight anatomical entity masks. For this purpose, we spatially normalized Zubal's MRI on the SPM T1 template using SPM [13. Since the SPM SPECT template is perfectly aligned with the SPM T1 template, the non-linear geometric transformation computed after spatial normalization was applied to our eight binary masks. In order to deal with partial volume effects in SPECT due to different spatial resolutions between SPECT and MRI, we smoothed our binary masks with a Gaussian kernel (full width at half maximum of $15 \mathrm{~mm}$ ). For each anatomical entity, perfusion measurements consisted in averaging the SPECT template intensities weighted by the smoothed masks. Note that in the white matter, regions of interest were delineated manually before SPECT template measurement, as the smoothed white matter mask included too much gray matter. As a result we generated an activity map mimicking normal brain perfusion.

Normal SPECT Simulation The attenuation and activity maps described above were used by PHG to simulate SPECT data. We chose to simulate photon emission of Technetium ${ }^{99 m}$ Tc. $10^{9}$ photons were simulated. A SPECT acquisition with a parallel hole collimator was simulated (64 projections 128 x 128 over $360^{\circ}$, pixel size $=2.2 \mathrm{~mm}$ ). Tomographic reconstruction used Filtered BackProjection with a ramp filter and the reconstructed data were postfiltered using a 3D Gaussian filter with full width at half maximum of $8.8 \mathrm{~mm}$. This provided us with a realistic, normal SPECT that was perfectly aligned (on account of its very construction) with a high resolution T1-weighted MRI.

\subsection{Application: MRI/SPECT Registration Validation}

In this section, we present an application of our simulation environment to validate MRI/SPECT registration methods in the case of non-pathological SPECT and MRI data sets. We decided to study and compare four registration methods based on statistical similarity measurements that are widely used in the context of automatic multimodality registration.

\section{Registration Methods Based on Statistical Similarity Measurements $\mathrm{SPECT} / \mathrm{MRI}$ registration is rigid intra-patient registration. The purpose is to}

$\overline{2}$ http://www.fil.ion.ucl.ac.uk/spm/ 
assess a rigid geometric $T$ transformation defined by six parameters (three translations and three rotations). Let the reference image $R$ be our SPECT data set and the floating image $F$ our MRI data set. Similarity measurement-based registration relies on the fact that a similarity measurement $S(R, T(F))$ is optimal when the data sets are perfectly registered. We studied four similarity measurements: Mutual Information, Normalised Mutual Information, Correlation Ratio and Woods Criterion.

- Mutual Information (MI) 7] [8]:

$$
S(A, B)=H(A)-H(A \mid B)=H(A)+H(B)-H(A, B)
$$

where $H$ denotes Shannon entropy.

Mutual Information is a measure of statistical dependence between two random variables, in our case the two images, relying on entropy measurements. Mutual Information makes no assumptions regarding the nature of this dependence.

- Normalised Mutual Information (NMI) [6]:

$$
S(A, B)=\frac{H(A)+H(B)}{H(A, B)}
$$

Normalised Mutual Information is also an entropy-based measure but invariant to the overlap region of both data sets.

- Correlation Ratio (CR) [9]:

$$
S(A \mid B)=\frac{\operatorname{Var}[E(A \mid B)]}{\operatorname{Var}(A)}
$$

Correlation Ratio measures the functional dependence between $\mathrm{A}$ and B, i.e. it measures how B explains the "energy" of A.

- Woods Criterion (WC) [4:

$$
S(A \mid B)=E_{B}\left(\frac{\sqrt{\operatorname{Var}(A \mid B)}}{E(A \mid B)}\right)
$$

Woods Criterion is based on the heuristic that certain structures or organs have a similar uniformity of intensities in both modalities. For each MRI intensity value, this criterion measures the ratio between standard deviation and mean of corresponding SPECT intensity values. As recommended by the author, we segmented brain from the MRI data set before registration.

Given a geometric $T$ transformation, each of these similarity measurements may be computed on the joint histogram of $R$ and $T(F)$. Partial volume interpolation was used to assess joint histograms. To avoid interpolation artifacts that occur when voxel dimensions of both data are multiples [14, we resampled our simulated SPECT data. MRI voxel dimensions being $1.1 \mathrm{~mm}$, we changed SPECT voxel dimensions from $2.2 \mathrm{~mm}$ to $4.51 \mathrm{~mm}$, which exactly corresponds 
to the sampling rate used for our clinical acquisitions. The optimization of these cost functions was then achieved using Powell's multidimensional direction set method and Brent's one-dimensional optimization algorithm for line minimizations [15]. A two-level multiresolution strategy as described by [8] was applied to avoid the pitfall of local optima.

Registration Validation $N=50 T^{*}$ theoretical transformations were generated by randomly sampling a 6 parameter vector using a Gaussian distribution $($ Mean $=0$, Standard Deviation $=10) . T^{*}$ was then applied to the MRI data set and a new unregistered MRI was thus created using trilinear interpolation during resampling. We then sequentially launched registration with the simulated SPECT using the four registration methods described above. We called the computed geometric transformation $\hat{T}$.

- Precision : For each registration method, precision was assessed by the distribution of $\left|T^{*}-\hat{T}\right|$ values.

- Accuracy : For each registration method, accuracy was assessed by computing mean, standard deviation and maximum values of a registration error measured on a set of n $P_{i}$ points uniformly distributed within the brain and on the skin (identified using labeled MRI) as follows :

$$
R M S=\sqrt{\frac{1}{n} \sum_{i=1}^{n}\left\|P_{i}-\hat{T}^{-1}\left(T^{*}\left(P_{i}\right)\right)\right\|^{2}}
$$

\section{Results}

Model Construction and SPECT Simulations Fig. 11 presents our normal brain perfusion model or activity map. After simulation, 22 million photons among the $10^{9}$ that were simulated were accepted by the detector. Volume reconstruction of this simulation is represented in Fig. 2 ,

Results of Registration Validation Distribution of precision measurements $\left(\left|T^{*}-\hat{T}\right|\right)$ are presented using boxplot representations for MI(Fig. 3), NMI(Fig. 4), CR(Fig. 5) and WC without (Fig. 6) or with (Fig. 7) prior brain segmentation from MRI. Translation errors $(T x, T y, T z)$ were computed in mm whereas rotation errors $(R x, R y, R z)$ were computed in degrees. We used the following reference coordinate system: $\mathrm{x}$ axis denotes anterior to posterior axis, $\mathrm{y}$ axis denotes top to bottom axis and $\mathrm{z}$ axis denotes left to right axis.

Accuracy measurements (mean, standard deviation and maximum values of RMS) are presented in Table[1, for $n=1600$ points uniformly distributed within the brain and $n=1400$ points uniformly distributed on the skin. RMS values are given in mm. Distribution of brain and skin RMS values for MI, NMI, CR and WC (with prior brain segmentation) is displayed using boxplot representations (Fig. 8). No registration solution was excluded from these results. 


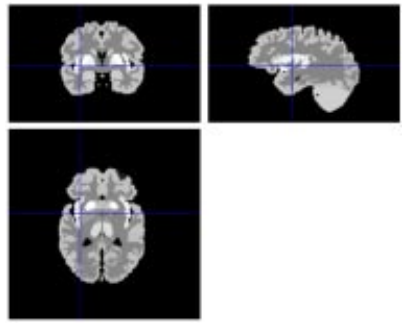

Fig. 1. Normal Brain Perfusion Model

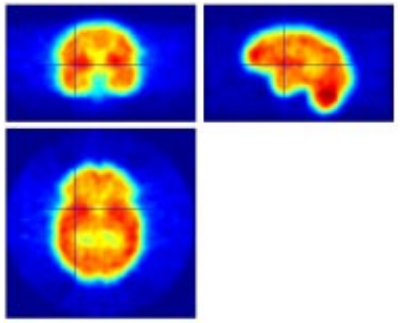

Fig. 2. Monte Carlo Simulations of Normal SPECT

Table 1. Accuracy Measurements

\begin{tabular}{|c|c|c|c|c|}
\hline & \multicolumn{2}{|c|}{ Brain RMS } & \multicolumn{2}{c|}{ Skin RMS } \\
\hline & Mean \pm Std & Max & Mean \pm Std & Max \\
\hline MI & $7.75 \pm 2.86$ & 21.02 & $8.67 \pm 3.27$ & 22.59 \\
\hline NMI & $8.13 \pm 2.97$ & 21.43 & $9.21 \pm 3.39$ & 23.17 \\
\hline CR & $5.60 \pm 2.83$ & 16.63 & $6.36 \pm 3.44$ & 18.05 \\
\hline WC (no segmented brain) & $19.87 \pm 5.26$ & 36.09 & $27.29 \pm 7.03$ & 50.74 \\
\hline WC (segmented brain) & $8.14 \pm 2.33$ & 16.57 & $9.07 \pm 2.95$ & 19.23 \\
\hline
\end{tabular}

\section{Discussion}

Thanks to the method we describe in this paper, we produced a realistic model of SPECT that was perfectly aligned with a T1-weighted MRI data set. The very concept of our approach provides a gold standard to validate MRI/SPECT registration methods. Simulated SPECT data sets were considered realistic because physical processes involved in SPECT acquisition were all closely modeled through PHG. Although our theoretical brain perfusion model does not take detailed physiological knowledge into account, we consider that our simulation results are adequate for registration validation purposes. What we present is a new way to evaluate and compare registration methods using realistic simulated data sets by controlling acquisition procedures (Monte Carlo simulations) and functional information (perfusion model).

Registration precision and accuracy proved very satisfactory for practically every method. Mean registration errors were lower than SPECT data resolution (resolution measurement: $12.4 \mathrm{~mm}$ ). Some of the less satisfactory results were found when assessing WC with unsegmented brain. This is not surprising since in this case uniformity assumptions made by the Woods Criterion are less valid. No significant differences were found between MI, NMI and WC (with segmented brain). Nevertheless, WC using segmented brain is less automated method, regardless of clinical use. The best results were obtained for CR. We are aware that our simulation approach may implicitly introduce statistical or functional 


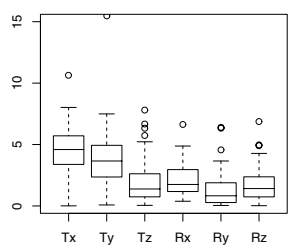

Fig. 3. MI Precision measurements $\left(\left|T^{*}-\hat{T}\right|\right)$

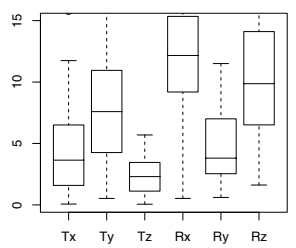

Fig. 6. WC Precision measurements $\left(\left|T^{*}-\hat{T}\right|\right)$ (no segmented brain)

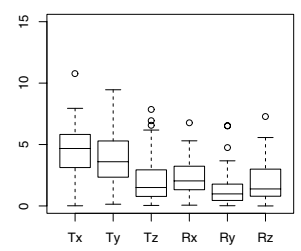

Fig. 4. NMI Precision measurements $\left(\left|T^{*}-\hat{T}\right|\right)$

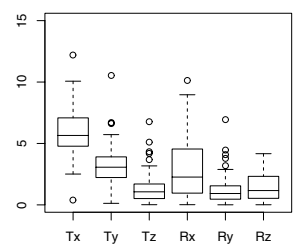

Fig. 7. WC Precision measurements $\left(\left|T^{*}-\hat{T}\right|\right)$ (segmented brain)

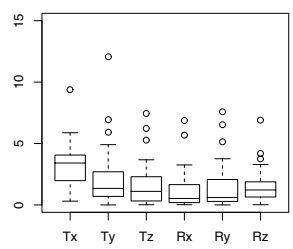

Fig. 5. CR Precision measurements $\left(\left|T^{*}-\hat{T}\right|\right)$

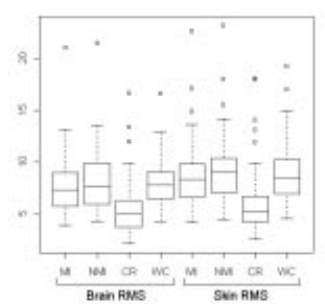

Fig. 8. Brain and skin accuracy measurements (RMS)

dependence between both data sets. Consequently, results may be better in our study than in real conditions. We plan to study the assumption when creating a pathological perfusion model. Still, our results are qualitatively concordant with literature [2], even though comparing different validation methods is not an easy thing.

We emphasize the generic aspect of our approach. Actually, this study may easily be extended to other registration methods or investigate other aspects such as sensitivity to SPECT reconstruction and correction features (e.g. reconstruction filters, attenuation or scatter correction). Our method may also be applied to study other clinical contexts, in particular by simulating pathological SPECT patterns. Our perfusion model could be improved, however, if it were based on the statistical analysis of a population of subjects (healthy or pathological) rather than on measurements from an averaged SPECT data set. For instance, we plan to study the behavior of the same similarity-based methods in the case of ictal SPECT in epileptic patients because of possibly large dissimilarities between ictal SPECT and MRI data.

\section{Acknowledgments}

This work was partly supported by a grant from the "Ligue Française Contre l'Epilepsie". 


\section{References}

1. West J., Fitzpatrick J. M., Wang M. Y., Dawant B. M., Maurer C. R. et al. Comparison and evaluation of retrospective intermodality brain image registration techniques. Journal of Computer Assisted Tomography, 21(4):554-566, 1997.

2. Barnden L., Kwiatek R., Lau Y., Hutton B., Thurjfell L., Pile K., and Rowe C. Validation of fully automatic brain SPET to MR co-registration. European Journal of Nuclear Medecine, 27(2):147-154, 2000.

3. Pfluger T., Vollmar C., Wismüller A., Dresel S., Berger F., Stuntheim P., Leisinger G., and Hahn K. Quantitative comparison of automatic and interactive methods for MRI-SPECT image registration of the brain based on 3-dimensional calculation of error. Journal of Nuclear Medecine, 41:1823-1829, 2000.

4. Woods R. P., Mazziotta J. C., and Cherry S. R. MRI-PET registration with automated algorithm. Journal of Computed Assisted Tomography, 17(4):536-546, 1993.

5. Ardekani B.A., Braun M., Hutton B.F., Kanno I., and Iida H. A fully automatic multimodality image registration algorithm. Journal of Computed Assisted Tomography, 19:615-623, 1995.

6. Studholme C., Hill D.L.G., and Hawkes D.J. An overlap invariant entropy measure of 3d medical image alignment. Pattern Recognition, 32:71-86, 1999.

7. Wells III W.M., Viola P., Atsumi H., Nakajima S., and Kikinis R. Multi-modal volume registration by maximization of mutual information. Medical Image Analysis, 1(1):35-51, 1996.

8. Maes F., Collignon A., Vandermeulen D., Marchal G., and Suetens P. Multimodality image registration by maximization of mutual information. IEEE Transactions on Medical Imaging, 16(2):187-198, 1997.

9. Roche A., Malandain G., Pennec X., and Ayache N. The correlation ratio as a new similarity measure for multimodal image registration. In MICCAI 98: Lecture Notes in Computer Science, 1496:1115-1124, 1998.

10. Harrison R.L., Vannoy S.D., Haynor D.R., Gillispie S.B., Kaplan M.S., and Lewellen T.K. Preliminary experience with the photon history generator module of a public-domain simulation system for emission tomography. In Conf. Rec. Nucl. Sci. Symp., 2:1154- 1158, 1993.

11. Zubal I.G., Harrell C.R., Smith E.O., Rattner Z., Gindi G.R., and Hoffer P.B. Computerized three-dimensional segmented human anatomy. Medical Physics, 21(2):299-302, 1994.

12. Kwiatek R., Barnden L., Tedman R., Jarret R., Chew J., Rowe C., and Pile K. Regional cerebral blood flow in fibromyalgia. Arthritis and Rheumatism, 43(12):28232833, 2000.

13. Friston K.J., Ashburner J., Poline J.B., Frith C.D., Heather J.D., and Frackowiak R.S.J. Spatial registration and normalization of images. Human Brain Mapping, 2:165-189, 1995.

14. Pluim J. P. W., Maintz J. B. A., and Viergever M. A. Interpolation artefacts in mutual information-based image registration. In Computer Vision and Image Understanding: CVIU, 77(2):211-232, 2000.

15. Press W. H., Teukolsky S. A., Vetterling W. T., and Flannery B. P. Numerical Recipes in C, 2nd. edition. Cambridge University Press, 1992. 